\title{
Modelo de simulação para estimar a infraestrutura necessária à assistência oncológica no sistema público de saúde
}

\author{
Saint Clair Santos Gomes Junior ${ }^{1}$ e Rosimary Terezinha Almeida²
}

Como citar

Gomes Junior SCS, Almeida RT. Modelo de simulação para estimar a infraestrutura necessária à assistência oncológica no sistema público de saúde. Rev Panam Salud Publica. 2009;25(2):113-9.

RESUMO Objetivo. Desenvolver um modelo de simulação para estimar a infraestrutura necessária à assistência oncológica no sistema público de saúde do Estado de São Paulo, Brasil, tendo por base dados de domínio público.

Método. Dados de domínio público do Sistema Único de Saúde referentes à produção assistencial de janeiro de 2002 a janeiro de 2004 para cirurgia oncológica, quimioterapia e radioterapia foram utilizados para estimar o número de casos de câncer no Estado. Os percentuais de cada procedimento terapêutico observados no Registro Hospitalar de Câncer foram combinados com os dados da produção assistencial para estimar um perfil de necessidade de serviço. Modelos de mistura foram utilizados para identificar subgrupos de casos de câncer com tempos de utilização diferenciados para quimioterapia e radioterapia. Um modelo de simulação foi empregado para estimar a infraestrutura necessária de acordo com os parâmetros definidos.

Resultados. O modelo estimou a necessidade de cirurgia para 52,5\% dos casos de câncer, radioterapia para $42,7 \%$ e quimioterapia para 48,5\%. Os modelos de mistura identificaram dois subgrupos de casos com tempo médio de utilização diferenciado para radioterapia e quatro subgrupos para quimioterapia. Com esses parâmetros, a infraestrutura estimada foi de: 147 salas de cirurgia, 2653 leitos cirúrgicos, 297 poltronas de quimioterapia e 102 equipamentos de terapia por radiação. Essas estimativas indicam a necessidade de aumentar os serviços de quimioterapia em 1,2 e os serviços de radioterapia em 2,4 vezes em relação aos parâmetros de programação utilizados atualmente no sistema público de saúde.

Conclusão. A utilização de modelos dessa natureza propicia uma melhor distribuição dos recursos em saúde, uma vez que toma como base a necessidade assistencial regional.

Palavras-chave Alocação de recursos, modelos matemáticos, planejamento em saúde, simulação, sistemas de informação, Brasil.

A base institucional do sistema público de saúde brasileiro é o Sistema Único de Saúde (SUS). Esse sistema se

1 Fundação Oswaldo Cruz, Instituto Fernandes Figueira, Unidade de Pesquisa Clínica. Correspondência: Avenida Rui Barbosa 716, CEP 22250-020, Rio de Janeiro, RJ, Brasil. Fone: +55-21-2554.1917; e-mail: scgomes@iff.fiocruz.br, scgomesjr@gmail.com

2 Universidade Federal do Rio de Janeiro, Coordenação dos Programas de Pós-Graduação em Engenharia, Programa de Engenharia Biomédica. E-mail: rosal@peb.ufrj.br caracteriza por uma rede de ações e serviços regionalizada e hierarquizada e tem como princípios a universalização do acesso e a integralidade das ações de saúde. De acordo com os princípios do SUS, a assistência oncológica deve ser oferecida de forma integral, com recursos para o diagnóstico definitivo, o estadiamento do tumor, o tratamento, a reabilitação e a paliação dos casos de câncer (1-3).
No SUS, a assistência oncológica integral é ofertada nos Centros de Alta Complexidade em Oncologia (CACON) (4). No ano de 2002, o sistema contava com 175 prestadores de serviço registrados como CACON no Cadastro Nacional de Estabelecimentos de Saúde (CNES) (5), sendo numericamente insuficiente, uma vez que o sistema deveria contar com pelo menos $320 \mathrm{CACON}$, tendo em vista a estimativa de 320 mil casos novos de 
câncer por ano e o parâmetro de programação da época de um CACON para cada 1000 casos novos de câncer por ano (6). Cabe destacar que um CACON deveria estar projetado para oferecer serviços de cirurgia, quimioterapia e radioterapia para 45,60 e $70 \%$ dos casos de câncer atendidos $(3,4)$.

Esses e outros parâmetros $(3,4,7)$ foram estimados numa época em que as bases de dados de produção do SUS apresentavam duplicidade de registro, impossibilidade de identificar cirurgias oncológicas e impossibilidade de recuperar o histórico de tratamento de um caso de câncer $(3,8)$. Esforços vêm sendo realizados no sentido de resolver parte dessas limitações. Contudo, verifica-se ainda a necessidade de uma metodologia que permita verificar e monitorar a aplicabilidade desses parâmetros a partir dos dados do SUS de domínio público $(3,8)$.

O objetivo do presente trabalho foi desenvolver um modelo de simulação que permitisse estimar os parâmetros de programação da assistência oncológica a partir dos dados do SUS de domínio público, assim como a infraestrutura necessária à assistência oncológica no Estado de São Paulo, Brasil.

\section{MATERIAIS E MÉTODOS}

O desenvolvimento deste trabalho teve quatro etapas básicas: a identificação dos casos de câncer nas bases de dados do SUS de domínio público; a identificação do perfil de necessidade terapêutica desses casos; a modelagem do tempo de utilização de cada modalidade terapêutica; e o dimensionamento da infraestrutura dos serviços de assistência oncológica para o Estado de São Paulo. O Estado de São Paulo foi selecionado por ser o único a disponibilizar, via Fundação Oncocentro de São Paulo (FOSP), dados dos Registros Hospitalares de Câncer (RHC) para todos os casos registrados e que receberam tratamento nos CACON do Estado (9).

\section{Identificação dos casos de câncer nas bases de dados do SUS}

As bases de dados do SUS de domínio público possibilitaram recuperar o histórico dos casos de câncer nas modalidades terapêuticas de quimioterapia, radioterapia e cirurgia. Não havia dados disponíveis sobre exames diagnósticos, reabilitação e paliação para os casos identificados.
Para as modalidades de quimioterapia e radioterapia, os casos de câncer foram identificados no módulo de oncologia do subsistema de Autorizações de Procedimentos de Alta Complexidade do Sistema de Informação Ambulatorial do SUS (APACSIA/SUS). Foi utilizada neste estudo toda a produção registrada no APACSIA/SUS para os procedimentos de quimioterapia e de radioterapia realizados no Estado de São Paulo no período de janeiro de 2002 a janeiro de 2004. A partir dessa base de dados, recuperou-se o histórico de tratamento de todos os casos de câncer cujo diagnóstico de tumor datava de 2002, os quais foram classificados nas seguintes modalidades terapêuticas: quimioterapia, radioterapia e quimioterapia combinada com radioterapia. A metodologia para a identificação de um caso de câncer no módulo de oncologia do APACSIA/SUS encontra-se descrita em trabalhos anteriores $(10,11)$.

Para a modalidade de cirurgia oncológica, os casos de câncer foram identificados no Sistema de Informações Hospitalares do SUS (SIH/SUS), utilizando-se toda a produção hospitalar registrada no período de janeiro de 2002 a janeiro de 2004 no Estado de São Paulo. A partir desses dados, realizou-se a identificação dos casos de câncer no SIH/SUS conforme descrito em trabalho anterior (7).

$\mathrm{O}$ SIH/SUS não registra a data de diagnóstico do tumor de um caso de câncer que se submeteu a cirurgia oncológica. Para tanto, estimou-se que 95\% dos casos de câncer que se submeteram a cirurgia oncológica em 2002 tiveram o diagnóstico no mesmo ano. Esse percentual corresponde à diferença observada entre a série histórica de casos de câncer do SIH/SUS por data de competência e a série histórica de casos de câncer do RHC/FOSP por data de diagnóstico.

\section{Perfil de necessidade de modalidades terapêuticas}

O perfil de necessidade reflete uma combinação das três modalidades terapêuticas identificadas nas bases de dados. Como essa combinação não pode ser obtida diretamente nas bases de produção (9), foi necessário estimar o perfil aplicando os percentuais observados no RHC/FOSP para cada modalidade terapêutica ao número de casos de câncer identificados nas bases de dados do SUS. Por exemplo, o módulo de oncologia do
APACSIA/SUS forneceu o número de casos de câncer na modalidade terapêutica de quimioterapia e quimioterapia combinada com radioterapia. Os casos de câncer nessas duas modalidades terapêuticas podem ou não ter se submetido a cirurgia oncológica. Para estimar o número de casos de câncer submetidos a cirurgia oncológica, procedeu-se da seguinte forma: 1) recuperou-se no RHC/ FOSP o percentual de casos de câncer com indicação de cirurgia oncológica nas modalidades terapêuticas de quimioterapia e quimioterapia combinada com cirurgia; 2) aplicou-se esse percentual ao número de casos de câncer identificados no módulo de oncologia do APACSIA/ SUS para a modalidade terapêutica de quimioterapia.

Assim, obteve-se uma estimativa do número de casos de câncer submetidos a cirurgia oncológica a partir dos casos de câncer de quimioterapia identificados no APACSIA/SUS. Esse processo foi repetido para todas as demais combinações de modalidades terapêuticas consideradas neste trabalho. O processo de estimação encontra-se descrito em trabalho anterior (8).

\section{Modelagem do tempo de utilização das modalidades terapêuticas}

A modelagem foi realizada utilizando modelos de mistura de probabilidade (12), que permitiram identificar grupos de casos de câncer com tempo diferenciado de utilização por modalidade terapêutica. A lógica de ajuste de um modelo de mistura consiste em combinar um número crescente de distribuições de probabilidade $(2,3,4, \ldots)$ até que, a partir de um determinado critério, verifique-se um ajuste adequado do modelo ao dado observado (13).

Os critérios para o ajuste adequado de um modelo de mistura podem ser visuais ou analíticos. Neste trabalho, foi utilizado o critério visual, dada a necessidade de se identificar o modelo com o ajuste mais próximo ao dado observado. No entanto, como critério de desempate, recorreu-se a um critério analítico, a saber, o critério de Gelfand e Ghosh (GGC) (14). As estimativas dos parâmetros dos modelos de mistura foram obtidas a partir do método bayesiano, que fornece dados mais precisos para a classe de modelos utilizada neste trabalho (15).

As distribuições normal e de Poisson foram utilizadas para o ajuste dos mode- 
los de mistura que melhor representassem o tempo de utilização dos serviços de quimioterapia e de radioterapia. Os parâmetros dessas distribuições foram estimados a partir do número de $\mathrm{Au}$ torizações de Procedimentos de Alta Complexidade em Oncologia (APACOncologia) que um caso de câncer apresentou ao longo do tratamento. $\mathrm{O}$ total de registros de APAC-Oncologia de um caso de câncer foi considerado como o tempo de tratamento em meses, uma vez que cada mês em tratamento gera um registro na base de dados $(8,11)$.

O tempo de utilização dos serviços de cirurgia oncológica foi ajustado a partir de uma mistura de distribuições beta, tradicionalmente utilizadas para representar fenômenos contínuos e delimitados em intervalos de tempo (16). O parâmetro dessa distribuição foi estimado a partir do tempo de permanência do caso de câncer no leito hospitalar, podendo variar de 1 a 365 dias. Esse intervalo de tempo foi definido como o parâmetro de programação dos serviços de cirurgia pela Portaria 1.101/ GM (17).

\section{Dimensionamento da infraestrutura dos serviços}

Um modelo de simulação foi utilizado para dimensionar a infraestrutura necessária dos serviços de assistência oncológica no SUS. A decisão de utilizar um modelo de simulação resultou da flexibilidade desse tipo de modelo para tratar cenários nos quais existe pouca informação quanto à precisão dos parâmetros utilizados (17). A lógica de desenvolvimento de um modelo de simulação consiste em reproduzir o funcionamento de um cenário por meio de um conjunto de distribuições de probabilidade (16); as modificações no cenário ocorrem a partir de alterações nos parâmetros das distribuições de probabilidade utilizadas $(18,19)$. Devido a essa característica, os modelos de simulação são considerados a ferramenta mais adequada para tratar os mais variados problemas de planejamento em saúde (20).

Os parâmetros utilizados no modelo de simulação foram número de casos de câncer por modalidade terapêutica, volume de procedimentos e infraestrutura dos serviços de assistência oncológica. $\mathrm{O}$ número de casos de câncer por modalidade terapêutica foi simulado por meio da distribuição de Bernoulli (16). A esti- mativa do parâmetro dessa distribuição foi obtida a partir do perfil de necessidade das modalidades terapêuticas. $\mathrm{O}$ segundo parâmetro, volume de procedimentos, foi obtido pela multiplicação do tempo de utilização de cada modalidade terapêutica (estimado pelos modelos de mistura) pelo número de casos de câncer simulados na etapa anterior.

O terceiro e último parâmetro, infraestrutura dos serviços de assistência oncológica, foi simulado a partir dos parâmetros de programação fornecidos pela Comissão Estadual de Reorganização, Reorientação e Acompanhamento da Assistência Oncológica do Rio de Janeiro (CERRAO) (21), a saber: 1) um serviço de cirurgia oncológica para cada 360 casos por ano, composto de uma sala de cirurgia para cada 180 cirurgias por ano e um leito cirúrgico para cada 10 internações por ano; 2) um serviço de quimioterapia para cada 600 casos por ano, composto de uma poltrona para cada 600 atendimentos por ano, um oncologista clínico e um técnico de enfermagem para cada 1800 atendimentos por ano e um profissional de enfermagem para cada 3600 atendimentos por ano; 3) um serviço de radioterapia para cada 500 casos por ano, composto de um equipamento de terapia por radiação, um médico radioterapeuta, um físico-médico e um profissional de enfermagem para cada 500 atendimentos por ano e um técnico de radioterapia para cada 250 atendimentos por ano.

A infraestrutura necessária dos serviços de assistência oncológica foi estimada pelo modelo de simulação considerando dois cenários:

- Cenário 1: utilização dos parâmetros de programação definidos na Portaria 741 /GM (22), que considera 6 meses de utilização para os serviços de quimioterapia, 1 mês para os serviços de radioterapia e 8 dias de permanência em leito hospitalar;

- Cenário 2: utilização dos parâmetros de programação estimados neste trabalho.

O pacote estatístico Statistical Analysis System $\left(\mathrm{SAS}^{\circledR}\right.$ ) versão 8.2 foi utilizado para a manipulação das bases de dados e para o desenvolvimento do modelo de simulação. As estimativas bayesianas e estatísticas de erro GGC dos modelos de mistura foram obtidas a partir do pacote estatístico WinBUGS ${ }^{\circledR}$ versão 4.0.

\section{RESULTADOS}

\section{Identificação dos casos de câncer nas bases de dados}

O módulo de oncologia do APACSIA/ SUS registrou 33266 casos de câncer com ano de diagnóstico do tumor em 2002. Desses, 13949 casos receberam tratamento de quimioterapia, 11357 receberam tratamento de radioterapia e 7960 receberam tratamento combinado de quimioterapia e radioterapia. No período de 2002 a 2004, esses casos de câncer geraram um total de 159372 APAC-Quimioterapia e 44959 APAC-Radioterapia. Com esses parâmetros, estima-se que o tempo médio de tratamento de um caso de câncer no Estado de São Paulo em quimioterapia seja de 7,3 meses, e em radioterapia, de 2,3 meses.

A partir dos dados de produção registrados no SIH/SUS para o ano de 2002, estimou-se que 16411 casos de câncer se submeteram a cirurgia oncológica no mesmo ano de diagnóstico do tumor.

\section{Estimação do perfil de necessidade das modalidades terapêuticas}

A tabela 1 apresenta a estimativa do número de casos de câncer submetidos a cirurgia oncológica no SUS, obtida a partir dos percentuais de tipo de tratamento observados no RHC/FOSP e da produção assistencial de quimioterapia, radioterapia e cirurgia oncológica observada nas bases da produção assistencial do SUS. Os casos de câncer na categoria "cirurgia combinada com outros tratamentos" foram desconsiderados, uma vez que, em tese, esses casos já estão contabilizados nas demais modalidades terapêuticas.

A tabela 2 apresenta a distribuição esperada de combinações de tratamento no sistema público de saúde para o Estado de São Paulo no ano de 2002 após o processo de estimação. As categorias "outras combinações de tratamento", "nenhum tratamento realizado" e "sem informação" foram obtidas diretamente do RHC/FOSP por não estarem disponíveis nas bases de dados do SIH/SUS e no módulo de oncologia do APACSIA/SUS.

Os casos de cirurgia oncológica, quimioterapia, radioterapia e suas combinações totalizaram 45210 casos de câncer (tabela 2). A partir desse número de casos, pode-se estimar que 23724 casos devem se submeter a uma cirurgia, 
TABELA 1. Resultado final do processo de estimação do número de casos de câncer submetidos a cirurgia a partir do número de casos identificados nas bases de dados do SUS de domínio público, Estado de São Paulo, Brasil, 2002

\begin{tabular}{|c|c|c|c|}
\hline $\begin{array}{l}\text { Combinações de } \\
\text { modalidade terapêutica } \\
\text { (No. de casos) }\end{array}$ & $\begin{array}{c}\text { No. de } \\
\text { casos no } \\
\text { RHC/FOSP }\end{array}$ & $\begin{array}{c}\% \text { de } \\
\text { casos no } \\
\text { RHC/FOSP }\end{array}$ & $\begin{array}{c}\text { No. de } \\
\text { casos de } \\
\text { câncer estimados }\end{array}$ \\
\hline \multicolumn{4}{|l|}{ Quimioterapia } \\
\hline Isolada & 3449 & 66,40 & 9263 \\
\hline Combinada com cirurgia & 1745 & 33,60 & 4686 \\
\hline Total & 5194 & 100,00 & 13949 \\
\hline \multicolumn{4}{|l|}{ Radioterapia } \\
\hline Isolada & 2375 & 66,36 & 7536 \\
\hline Combinada com cirurgia & 1204 & 33,64 & 3821 \\
\hline Total & 3579 & 100,00 & 11357 \\
\hline \multicolumn{4}{|l|}{ Radioterapia e quimioterapia } \\
\hline Isolada & 1854 & 58,88 & 4687 \\
\hline Combinada com cirurgia & 1295 & 41,12 & 3273 \\
\hline Total & 3149 & 100,00 & 7960 \\
\hline \multicolumn{4}{|l|}{ Cirurgia } \\
\hline Isolada & 11350 & 72,78 & 11944 \\
\hline Combinada com outros tratamentos & 4244 & 27,22 & 4467 \\
\hline Total & 15594 & 100,00 & 16411 \\
\hline
\end{tabular}

TABELA 2. Distribuição esperada de modalidades terapêuticas e suas combinações para os casos de câncer identificados nas bases de dados do SUS de domínio público, Estado de São Paulo, Brasil, 2002

\begin{tabular}{lc}
\hline \multicolumn{1}{c}{\begin{tabular}{c} 
Modalidades terapêuticas \\
\multicolumn{1}{c}{ e combinações }
\end{tabular}} & $\begin{array}{c}\text { Estimativas de casos de câncer } \\
\text { No. (\%) }\end{array}$ \\
\hline Cirurgia & $11944(24)$ \\
Radioterapia & $7536(15)$ \\
Quimioterapia & $9263(18)$ \\
Cirurgia e radioterapia & $3821(8)$ \\
Cirurgia e quimioterapia & $4686(9)$ \\
Radioterapia e quimioterapia & $4687(9)$ \\
Cirurgia, radioterapia e quimioterapia & $3273(6)$ \\
Subtotal & $45210(89)$ \\
Outras combinações de tratamento & $2307(5)$ \\
Nenhum tratamento realizado & $2895(6)$ \\
Sem informação & $188(0)$ \\
Total & $50600(100)$ \\
\hline
\end{tabular}

19317 casos devem receber radioterapia e 21909 casos devem receber quimioterapia no SUS em algum momento do tratamento, resultando em um perfil de necessidade de serviço de 52,5, 42,7 e $48,5 \%$, respectivamente.

\section{Modelagem do tempo de utilização das modalidades terapêuticas}

Os modelos de mistura forneceram a estimativa do tempo de utilização dos serviços de quimioterapia e radioterapia. O modelo com quatro distribuições normais foi o que apresentou o melhor ajuste visual para o tempo de utilização da modalidade terapêutica de quimioterapia isolada (figura 1A) e menor índice de estatística de erro GGC $(11,59)$. Os parâme- tros do modelo de melhor ajuste visual indicaram que 40,4\% dos casos de câncer receberam tratamento de quimioterapia por 2,4 meses, $25,0 \%$ por 5,7 meses, $11,2 \%$ por 8,5 meses e $23,4 \%$ receberam tratamento por 13,5 meses (tabela 3 ).

O modelo de mistura com oito distribuições de Poisson foi o que apresentou o menor índice de estatística de erro GGC $(16,83)$ para a modalidade terapêutica de quimioterapia combinada. No entanto, o melhor ajuste visual foi observado com uma mistura de três distribuições normais (figura 1B). Os parâmetros desse modelo indicaram que $23,8 \%$ dos casos de câncer receberam tratamento por 2,8 meses, $18,6 \%$ por 5,7 meses e $57,6 \%$ receberam tratamento por 11,4 meses (tabela 3).
O modelo de mistura com seis distribuições normais foi o que apresentou o menor índice de estatística de erro GGC $(2,10)$ para a modalidade terapêutica de radioterapia isolada. No entanto, o melhor ajuste visual para essa modalidade foi observado com uma mistura de duas distribuições normais (figura 1C). Os parâmetros desse modelo indicaram que 90,0\% dos casos de câncer receberam tratamento por 2,0 meses e $10,0 \%$ receberam tratamento por 4,0 meses (tabela 3 ).

O modelo de mistura de duas distribuições normais foi o que apresentou o menor índice de estatística de erro GGC $(343,00)$ e também o melhor ajuste visual para a modalidade terapêutica de radioterapia combinada (figura 1D). Os parâmetros desse modelo indicaram que 96,1\% dos casos de câncer receberam tratamento por 2,4 meses e 3,9\% receberam tratamento por 6,0 meses (tabela 3).

Finalmente, o melhor ajuste visual para o tempo de utilização dos serviços de cirurgia oncológica foi obtido a partir de um modelo beta univariado (figura 1E), utilizando-se os seguintes parâmetros: 0,54, 25,80, 1 e 365 . Os parâmetros utilizados forneceram uma estimativa de aproximadamente 8,0 dias para o tempo de utilização desse serviço (tabela 3 ).

\section{Estimação do volume de atendimentos}

O modelo de simulação retornou 24506 casos de câncer na modalidade terapêutica de quimioterapia, 21572 casos na modalidade de radioterapia e 26529 casos na modalidade de cirurgia oncológica (tabela 3). Multiplicando o número de casos simulados pelos tempos de utilização estimados, obteve-se o seguinte:

- Cenário 1: 147036 atendimentos de quimioterapia, 21572 atendimentos de radioterapia e 26529 atendimentos de cirurgia;

- Cenário 2: 178043 atendimentos de quimioterapia, 51002 atendimentos de radioterapia e 26529 atendimentos de cirurgia (tabela 3).

\section{Simulação da infraestrutura}

Os volumes de atendimentos foram aplicados aos parâmetros de programação fornecidos pela CERRAO para a obtenção da infraestrutura necessária aos serviços de assistência oncológica 
TABELA 3. Simulação do número de casos novos de câncer por ano e do volume de atendimentos gerados por modalidade terapêutica e tempo de utilização, Estado de São Paulo, Brasil, 2002

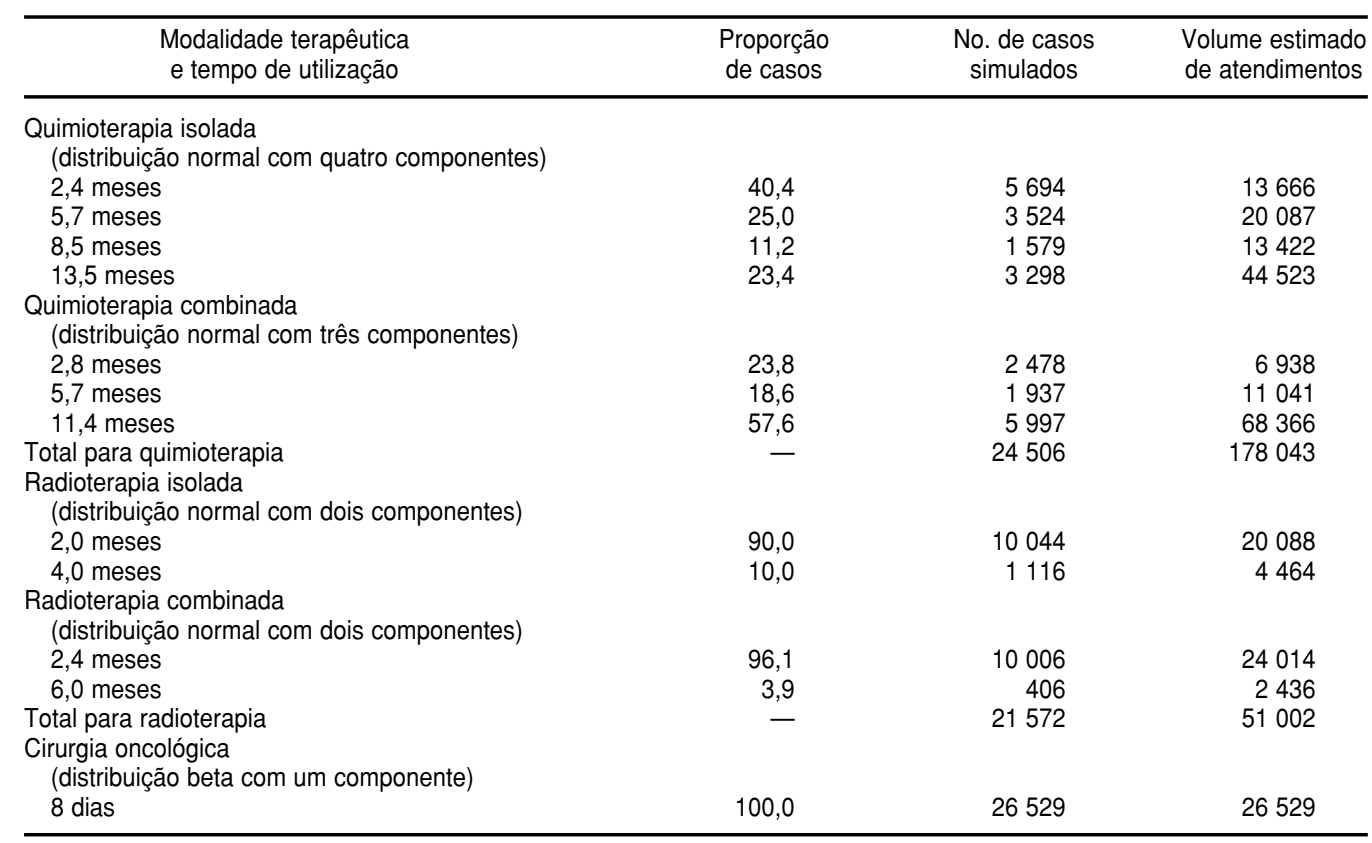

FIGURA 1. Resultado final da modelagem do tempo de utilização das modalidades terapêuticas identificadas nas bases de dados do SUS de domínio público, Estado de São Paulo, Brasil, 2002 ${ }^{\mathrm{a}}$
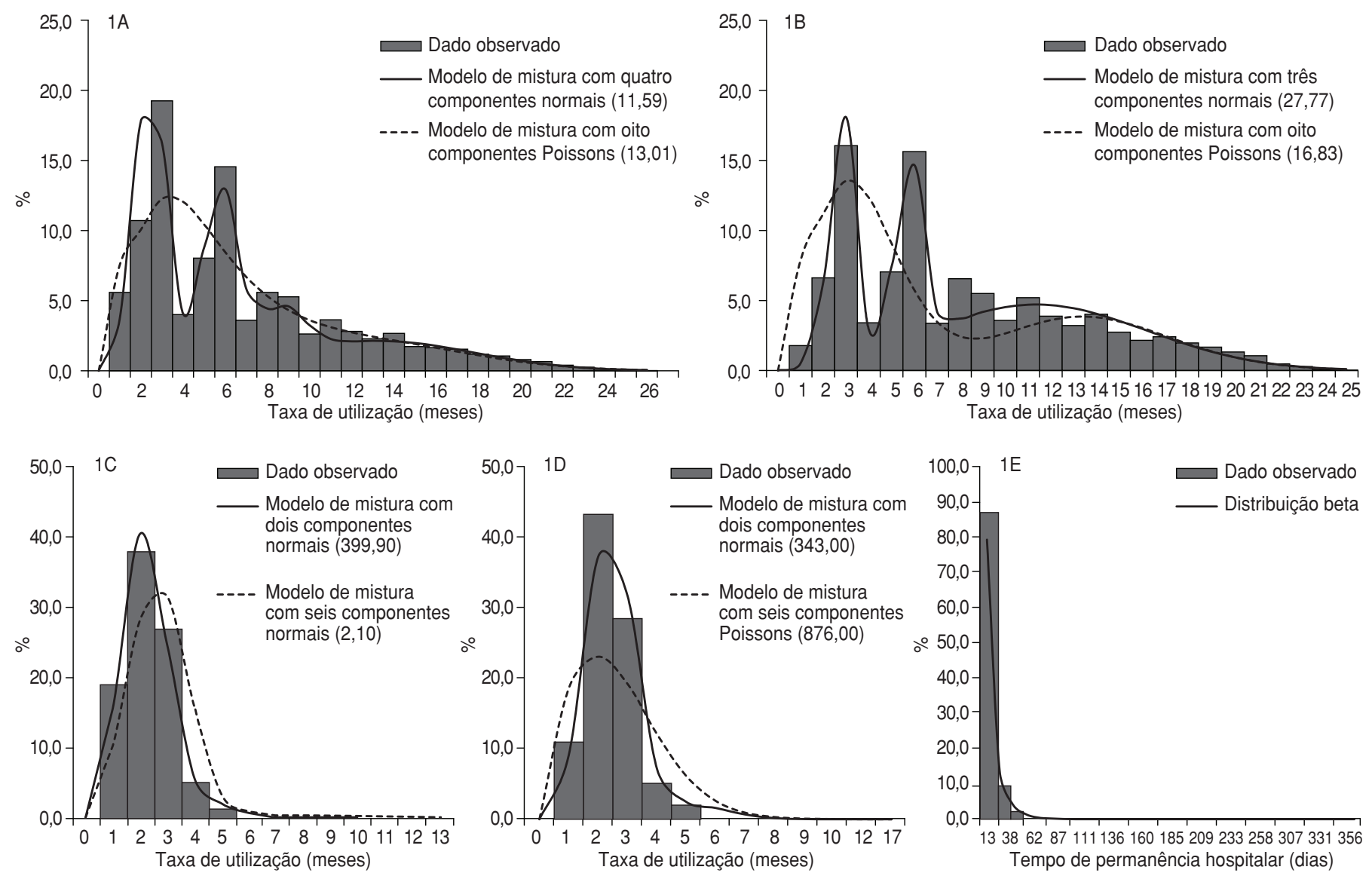

a A) Quimioterapia isolada; B) quimioterapia combinada; C) radioterapia isolada; D) radioterapia combinada; E) cirurgia. 
TABELA 4. Comparação da infraestrutura estimada por modalidade terapêutica para os dois cenários, Estado de São Paulo, Brasil, 2002

\begin{tabular}{lrr}
\hline \multicolumn{1}{c}{ Infraestrutura (No. de casos) } & Cenário & Cenário 2 \\
\hline Número de unidades CACON (50 600) & 51 & 51 \\
Cirurgia oncológica (26 529) & & \\
$\quad$ Salas de cirurgia & 2653 & 147 \\
Leitos cirúrgicos & & 2653 \\
Quimioterapia (24 506) & 245 & 297 \\
Poltronas & 82 & 99 \\
Oncologistas clínicos & 82 & 99 \\
Técnicos de enfermagem & 41 & 49 \\
Profissionais de enfermagem & & 102 \\
Radioterapia (21 572) & 43 & 102 \\
Equipamentos de terapia por radiação & 43 & 102 \\
Médicos radioterapeutas & 43 & 204 \\
Físico-médicos & 86 & 102 \\
Técnicos de radioterapia & 43 & \\
Profissionais de enfermagem & & \\
\hline
\end{tabular}

para cada um dos cenários considerados. Na comparação do cenário 2 (tempos de utilização por grupos de casos) com o cenário 1 (tempo de utilização fixo), observou-se a necessidade de aumentar os serviços de quimioterapia em 1,2 e os serviços de radioterapia em 2,4 vezes (tabela 4). A infraestrutura para os serviços de cirurgia oncológica não se alterou para os dois cenários, uma vez que não foram observadas diferenças quanto aos tempos de utilização estimados neste trabalho e aqueles definidos pela Portaria 1.101/GM (7).

\section{DISCUSSÃO}

O presente trabalho foi motivado tanto pelo maior volume de dados disponíveis atualmente nas bases de dados do SUS quanto pelas críticas referentes à necessidade de aprimorar os parâmetros de programação dos serviços de assistência oncológica (3). Os grandes desafios foram a obtenção de dados relativos ao perfil de necessidade das modalidades terapêuticas e o desenvolvimento de uma metodologia para a identificação de subgrupos de casos de câncer com base no tempo de utilização dessas modalidades.

Com relação ao perfil de necessidade das modalidades terapêuticas, acreditase que as estimativas obtidas para as modalidades de quimioterapia e radioterapia estejam próximas à realidade do SUS. O Estado de São Paulo apresenta um grande contingente de serviços privados, os quais têm como característica um registro mais adequado da produção desses procedimentos no módulo de on- cologia do APACSIA/SUS, a fim de receberem pelos serviços prestados (8). Por outro lado, acredita-se que as estimativas obtidas para cirurgia oncológica estejam subestimadas, em virtude da impossibilidade de recuperar o número de casos de câncer que foram tratados apenas com cirurgia oncológica fora dos CACON.

O segundo parâmetro considerado foi o tempo de utilização dos serviços, o qual foi associado ao perfil de necessidade das modalidades terapêuticas para fornecer uma estimativa do volume de procedimentos necessários para tratar os casos de câncer. Este trabalho utilizou modelos de mistura para estimar o tempo de utilização dos serviços nos subgrupos de casos de câncer identificados. Os modelos de mistura discriminaram, por exemplo, um subgrupo de casos de câncer com tempo de utilização dos serviços de quimioterapia superior a 10 meses, achado típico em casos tratados por hormonioterapia.

A modelagem do tempo de utilização ocorreu com base em críticas realizadas $(1,3)$ quanto à necessidade de estimar o tempo de utilização dos serviços de assistência oncológica por subgrupos de casos de câncer, já que a utilização de um valor fixo tende a subestimar a necessidade de casos que demandam tratamento por um tempo superior ao definido pelo parâmetro de programação. Os modelos de mistura, além de identificar tempos de utilização dos serviços por subgrupos de casos de câncer, também forneceram a variabilidade associada ao parâmetro, permitindo a realização de análises de sensibilidade.
A simulação da infraestrutura indicou uma necessidade de aumento de 4,6 vezes na atual oferta de leitos de cirurgia oncológica no Estado de São Paulo para garantir assistência integral a todos os casos de câncer que buscam atendimento no SUS; no ano de 2002, o Estado de São Paulo tinha 580 leitos cadastrados para o SUS (5), ao passo que o modelo de simulação revelou a necessidade de 2653 leitos. Cabe destacar que esse aumento parece razoável ao se considerar que parte das cirurgias oncológicas realizadas em hospitais gerais deveriam ser realizadas em CACON. Para a radioterapia, foi estimada uma necessidade total de 43 equipamentos no cenário 1 , contra 102 equipamentos no cenário 2 . As estimativas obtidas no cenário 2 encontramse mais próximas da realidade observada no Estado de São Paulo, que possuía cadastrado para o SUS um total de 206 equipamentos para terapia por radiação (5). Tal diferença pode estar denotando uma folga de 104 equipamentos, especialmente ao se considerar o parâmetro de programação fornecido pela CERRAO de 500 atendimentos por ano por equipamento.

$\mathrm{O}$ exemplo da radioterapia mostra a potencialidade do modelo de simulação em auxiliar o gestor de saúde na alocação de recursos: pode-se simular uma situação desejada e verificar o quanto de recurso deve ser programado para que tal situação seja alcançada. Destaca-se, ainda, a possibilidade de utilização do modelo para outros cenários, tendo sempre em conta as limitações regionais quanto à confiabilidade do registro de produção e a existência e disponibilidade de dados de um RHC. Em algumas realidades, a inexistência de um RHC local pode ser contornada utilizando-se os dados do RHC de uma localidade com perfil epidemiológico semelhante.

Como se pôde observar no presente estudo, o modelo de simulação é uma ferramenta útil no apoio ao complexo processo de decisão envolvido na programação dos serviços de assistência oncológica. Foi apresentada uma metodologia que permitiu aprimorar os parâmetros de programação até então utilizados. Espera-se que a proposta metodológica de aprimoramento das estimativas aqui apresentadas propicie um melhor ajuste na distribuição dos recursos destinados a assistência oncológica, passando a ter como ponto de partida o perfil de necessidade regional. 


\section{REFERÊNCIAS}

1. Kligerman J. Ampliação da assistência oncológica no Brasil. Rev Bras Cancerol. 2000;46 (4):347-9.

2. Kligerman J. Registro hospitalar de câncer no Brasil. Rev Bras Cancerol. 2001;47(4):357-9.

3. Gadelha MIP. Planejamento da assistência oncológica: um exercício de estimativas. Rev Bras Cancerol. 2002;48(4):533-43.

4. Marques CP. Projeto de expansão da assistência oncológica: EXPANDE, um novo modelo assistencial [dissertação]. Rio de Janeiro: Universidade do Estado do Rio de Janeiro; 2002.

5. Brasil, Ministério da Saúde, Secretaria de Atenção à Saúde, DATASUS. Cadastro nacional de estabelecimentos de saúde. Brasília: Ministério da Saúde. Disponível em: http:/ / cnes.datasus.gov.br. Acessado em 19 de junho de 2005.

6. Brasil, Ministério da Saúde, Secretaria de Atenção à Saúde. Relatório de gestão 2007. Brasîlia: Ministério da Saúde. Disponível em: http:/ / dtr2001.saude.gov.br/sas/relatorio/ 6.2\%20redes\%20assistenciais.htm\#6.2.7. Acessado em 23 de março de 2007.

7. Brasil, Ministério da Saúde. Portaria 1.101/ GM, de 12 de junho de 2002. Estabelece os parâmetros de cobertura assistencial no âmbito do Sistema Único de Saúde. Brasília: Ministério da Saúde. Disponível em: http:// dtr2001.saude.gov.br/sas/PORTARIAS/Port 2002/Gm/GM-1101.htm. Acessado em 23 de março de 2007.

8. Gomes Jr SCS. Modelo de simulação da infraestrutura necessária à assistência oncológica no Sistema Único de Saúde [tese]. Rio de Janeiro: Universidade Federal do Rio de Ja- neiro; 2005. Disponível em: http://bvsms. saude.gov.br/bvs/publicacoes/premio2006/ Saint_Clair_D_P.pdf. Acessado em 23 de março de 2007.

9. Fundação Oncocentro de São Paulo. Registro hospitalar de câncer no Estado de São Paulo - 2000. Cadernos FOSP. 2001;3.

10. Gomes Jr SCS, De Martino R, Almeida RT. Rotinas de integração das tabelas do sistema de autorização de procedimentos de alta complexidade em oncologia do Sistema Único de Saúde. Cad Saude Coletiva. 2003;11(2):231-54.

11. Gomes Jr SCS, Almeida RT. Identificação de um caso novo de câncer no Sistema de Informação Ambulatorial do Sistema Único de Saúde. Cad Saude Coletiva. 2004;11(1):57-68.

12. Richardson S, Green PJ. On Bayesian analysis of mixture with an unknown number of components. J Roy Stat Soc B. 1996;59(4):731-92.

13. Robert CP. Mixtures of distributions: inference and estimation. Em: Gilks WR, Richardson S, Spiegelhalter DJ. Markov chain Monte Carlo in practice. Londres: Chapman \& Hall; 1996. Pp. 441-61.

14. Ghosh S, Norris JL. Bayesian capturerecapture analysis and model selection allowing for heterogeneity and behavioral effects. J Agric Biol Environ Stat. 2005;10(1):35-49.

15. Yang CC, Muthén BO, Yang CC. Finite mixture multivariate generalized linear models using Gibbs sampling and E-M algorithms. Proc Natl Sci Counc ROC (A). 1999;23(6): 695-702.

16. Fishman GS. Discrete-event simulation: modeling, programming and analysis. Berlim: Springer Verlag; 2001.
17. Saliby E. Coleção COPPEAD de administração: repensando a simulação — a amostragem descritiva. São Paulo: Atlas; 1989.

18. Soares LFG. Modelagem e simulação discreta de sistemas. Rio de Janeiro: Campus; 1992.

19. Pidd M. Modelagem empresarial: ferramentas para tomada de decisão. Porto Alegre: Bookman; 1998.

20. Largegren $\mathrm{M}$. What is the role and contribution of models to management and research in the health services? A view from Europe. Eur J Oper Res. 1998;105(2):257-66.

21. Comissão Estadual de Reorganização, Reorientação e Acompanhamento da Assistência Oncológica do Rio de Janeiro. Diretrizes para reorganização, reorientação e acompanhamento da assistência oncológica no Estado do Rio de Janeiro. Rev Bras Cancerol. 2001;47(2):125-41.

22. Brasil, Ministério da Saúde. Portaria $741 /$ GM, de 19 de dezembro de 2005. Define as unidades de assistência de alta complexidade em oncologia, os centros de assistência de alta complexidade em oncologia (CACON) e os centros de referência de alta complexidade em oncologia e suas aptidões e qualidades. Brasília: Ministério da Saúde. Disponível em: http://dtr2001.saude.gov.br/sas/PORTARIAS/ Port2005/PT-741.htm. Acessado em 23 de março de 2007.

Manuscrito recebido em 3 de abril de 2007. Aceito em versão revisada em 6 de fevereiro de 2008.

ABSTRACT Objective. To develop a simulation model using public data to estimate the cancer care infrastructure required by the public health system in the state of São Paulo, Brazil. Method. Public data from the Unified Health System database regarding cancer surgery, chemotherapy, and radiation therapy, from January 2002-January 2004, were used to estimate the number of cancer cases in the state. The percentages recorded for each therapy in the Hospital Cancer Registry of Brazil were combined with the data collected from the database to estimate the need for services. Mixture models were used to identify subgroups of cancer cases with regard to the length of time that chemotherapy and radiation therapy were required. A simulation model was used to estimate the infrastructure required taking these parameters into account.

Results. The model indicated the need for surgery in $52.5 \%$ of the cases, radiation therapy in $42.7 \%$, and chemotherapy in $48.5 \%$. The mixture models identified two subgroups for radiation therapy and four subgroups for chemotherapy with regard to mean usage time for each. These parameters allowed the following estimated infrastructure needs to be made: 147 operating rooms, 2653 operating beds, 297 chemotherapy chairs, and 102 radiation therapy devices. These estimates suggest the need for a 1.2-fold increase in the number of chemotherapy services and a 2.4-fold increase in the number of radiation therapy services when compared with the parameters currently used by the public health system.

Conclusion. A simulation model, such as the one used in the present study, permits better distribution of health care resources because it is based on specific, local needs.

Key words Resource allocation, mathematical models, health planning, simulation, information systems, Brazil. 